\title{
Yuki Tsukamoto*
}

\section{Prescribed mean curvature equation on torus}

https://doi.org/10.1515/anly-2020-0054

Received November 12, 2020; accepted April 1, 2021

\begin{abstract}
Prescribed mean curvature problems on the torus have been considered in one dimension. In this paper, we prove the existence of a graph on the $n$-dimensional torus $\mathbb{T}^{n}$, the mean curvature vector of which equals the normal component of a given vector field satisfying suitable conditions for a Sobolev norm, the integrated value, and monotonicity.
\end{abstract}

Keywords: Prescribed mean curvature, fixed-point theorem

MSC 2010: 35J93

\section{Introduction}

In this paper, we consider the following prescribed mean curvature problem on the torus $\mathbb{T}^{n}:=\mathbb{R}^{n} / \mathbb{Z}^{n}$ :

$$
-\operatorname{div}\left(\frac{\nabla u}{\sqrt{1+|\nabla u|^{2}}}\right)=v(\nabla u) \cdot g(x, u(x)) \quad \text { on } \mathbb{T}^{n},
$$

where $v$ is the unit normal vector of $u$, that is,

$$
v(z)=\frac{1}{\sqrt{1+|z|^{2}}}(-z, 1) .
$$

The vector field $g\left(x, x^{n+1}\right): \mathbb{T}^{n} \times \mathbb{R} \rightarrow \mathbb{R}^{n+1}$ is given, and we seek a solution $u$ satisfying (1.1). The left-hand side of (1.1) represents the mean curvature of the graph of $u$, and the right-hand side is the normal component of the vector field $g$ on the graph.

In the case of Dirichlet conditions of a bounded domain $\Omega \subset \mathbb{R}^{n}$, prescribed mean curvature problems have been studied by numerous researchers. Bergner [3] solved the Dirichlet problem in the case where the right-hand side of (1.1) is $H=H(x, u, v(\nabla u))$ under the assumptions of boundedness $(|H|<\infty)$, monotonicity $\left(\partial_{n+1} H \geq 0\right.$ ), and convexity of $\Omega$. Under the same conditions for the function $H$, Marquardt [9] imposed a condition on $\partial \Omega$ depending on $H$ that guarantees the existence of solutions even for a domain $\Omega$ that is not necessarily convex. In [13], we proved the existence of a solution only under the condition that the Sobolev norm of $H$ is sufficiently small. In the case of a compact Riemannian manifold, Aubin [2] solved the linear elliptic problem $-\partial_{i}\left[a_{i j}(x) \partial_{j} u\right]=H(x)$ if the integrated value of $H$ is zero. The assumption of the integrated value plays an important role in the existence of solutions to elliptic equations on a compact Riemannian manifold. Denny [4] solved the quasilinear elliptic problem $-\operatorname{div}(a(u(x)) \nabla u)=H(x)$ on the torus $\mathbb{T}^{n}$ with $n=2$, 3. Prescribed mean curvature problems on the one-dimensional torus

$$
\left(\frac{u^{\prime}}{\sqrt{1+\left(u^{\prime}\right)^{2}}}\right)^{\prime}=H\left(x, u, u^{\prime}\right)
$$

have been investigated for a wide variety of conditions $H$ (we refer to, for example, $[5,7,8,10,11,14]$ ).

As we noted in [13], the motivation for the present study comes from a singular perturbation problem, and we proved the following in [12]. Suppose a constant $\varepsilon>0$ and functions $\phi_{\varepsilon} \in W^{1,2}$ and $g_{\varepsilon} \in W^{1, p}$,

*Corresponding author: Yuki Tsukamoto, Department of Mathematics, Tokyo Institute of Technology, 152-8551, Tokyo, Japan, e-mail: tsukamoto.y.ag@m.titech.ac.jp 
with $p>\frac{n+1}{2}$, satisfy

$$
\begin{aligned}
-\varepsilon \Delta \phi_{\varepsilon}+\frac{W^{\prime}\left(\phi_{\varepsilon}\right)}{\varepsilon} & =\varepsilon \nabla \phi_{\varepsilon} \cdot g_{\varepsilon}, \\
\int\left(\frac{\varepsilon\left|\nabla \phi_{\varepsilon}\right|^{2}}{2}+\frac{W\left(\phi_{\varepsilon}\right)}{\varepsilon}\right) d x+\left\|g_{\varepsilon}\right\|_{W^{1, p}(\tilde{\Omega})} & \leq C,
\end{aligned}
$$

where $W$ is a double-well potential such as $W(\phi)=\left(1-\phi^{2}\right)^{2}$. Then the interface $\left\{\phi_{\varepsilon}=0\right\}$ converges locally in the Hausdorff distance to a surface having a mean curvature given by $v \cdot g$ as $\varepsilon \rightarrow 0$. Here, $v$ is the unit normal vector of the surface, and $g$ is the weak $W^{1, p}$ limit of $g_{\varepsilon}$. If the surface is represented locally as a graph of a function $u$ on $\mathbb{T}^{n}$, we can observe that $u$ satisfies (1.1). In this paper, we prove the existence of solutions to (1.1) assuming that the Sobolev norm of $g$ is sufficiently small, $g^{n+1}$ for the $(n+1)$-st component is monotonous, and the integrated value of $g^{n+1}$ is zero. The following theorem is the main result.

Theorem 1.1. Fix $\frac{n+1}{2}<p<n+1$ and $q=\frac{n p}{n+1-p}$. Then there exists a constant $\varepsilon_{1}=\varepsilon_{1}(n, p)>0$ with the following property: If $\varepsilon<\varepsilon_{1}$, and

$$
g=\left(g^{1}, \ldots, g^{n}, g^{n+1}\right)=\left(g^{\prime}, g^{n+1}\right) \in W^{1, p}\left(\mathbb{T}^{n} \times(-1,1) ; \mathbb{R}^{n+1}\right)
$$

satisfies the relations

$$
\begin{aligned}
\|g\|_{W^{1, p}\left(\mathbb{T}^{n} \times(-1,1)\right)} & <\varepsilon^{\frac{2}{3}}, \\
\partial_{n+1} g^{n+1}\left(x, x^{n+1}\right) & >\varepsilon+\varepsilon^{\frac{1}{2}}\left|\partial_{n+1} g^{\prime}\left(x, x^{n+1}\right)\right|, \\
\int_{\mathbb{T}^{n}} g^{n+1}(x, 0) & =0,
\end{aligned}
$$

then there exists a function $u \in W^{2, q}\left(\mathbb{T}^{n}\right)$ such that

$$
-\operatorname{div}\left(\frac{\nabla u}{\sqrt{1+|\nabla u|^{2}}}\right)=v(\nabla u) \cdot g(x, u(x)) \quad \text { on } \mathbb{T}^{n} .
$$

Moreover, the following inequality holds:

$$
\left\|u-\int_{\mathbb{T}^{n}} u(y) d y\right\|_{W^{2, q}\left(\mathbb{T}^{n}\right)} \leq \varepsilon^{\frac{1}{2}} .
$$

Assumptions (1.2) and (1.3) guarantee the existence and uniqueness of solutions to the linearized problem of (1.1) where a given function depends on $\nabla u$. Equation (1.4) is necessary for the existence of solutions to elliptic equations on the torus. To the best of our knowledge, prescribed mean curvature problems on the torus in the general dimension have been insufficiently studied. However, we have proved the existence of the solution under natural assumptions.

The following is the method of proof. First, we find the conditions of $H$ for the linearized problem of (1.1), i.e.

$$
-\operatorname{div}\left(\frac{\nabla u}{\sqrt{1+|\nabla v|^{2}}}\right)=H,
$$

to have a unique solution. If we add a suitable constant term for any $v$, the function $v(\nabla v) \cdot g(x, v(x))$ satisfies the conditions. By estimating the norm of this solution with $g$, the mapping $T(v)=u$ has a fixed point using a fixed-point theorem, and Theorem 1.1 follows.

\section{Proof of Theorem 1.1}

A theorem that holds in the Euclidean space also holds on a torus, as we consider a function on a torus to be a periodic function in the Euclidean space.

Let $X\left(\mathbb{T}^{n}\right)$ be a function space on $\mathbb{T}^{n}$. We define a subspace $X_{\text {ave }}\left(\mathbb{T}^{n}\right) \subset X\left(\mathbb{T}^{n}\right)$ as

$$
X_{\text {ave }}:=\left\{w \in X: \int_{\mathbb{T}^{n}} w=0\right\} .
$$


Theorem 2.1. Suppose $v \in C^{1}\left(\mathbb{T}^{n}\right)$ and $H \in L_{\text {ave }}^{2}\left(\mathbb{T}^{n}\right)$. Then there exists a unique function $u \in W_{\text {ave }}^{1,2}\left(\mathbb{T}^{n}\right)$ such that

$$
\int_{\mathbb{T}^{n}} \frac{\nabla u \cdot \nabla \phi}{\sqrt{1+|\nabla v|^{2}}}=\int_{\mathbb{T}^{n}} H \phi
$$

for all $\phi \in W^{1,2}\left(\mathbb{T}^{n}\right)$.

Proof. We define a function $B: W_{\text {ave }}^{1,2}\left(\mathbb{T}^{n}\right) \times W_{\text {ave }}^{1,2}\left(\mathbb{T}^{n}\right) \rightarrow \mathbb{R}$ by

$$
B\left[w_{1}, w_{2}, v\right]:=\int_{\mathbb{T}^{n}} \frac{\nabla w_{1} \cdot \nabla w_{2}}{\sqrt{1+|\nabla v|^{2}}} .
$$

By the Hölder inequality, we obtain

$$
\begin{aligned}
\left|B\left[w_{1}, w_{2}, v\right]\right| & \leq \int_{\mathbb{T}^{n}}\left|\nabla w_{1}\right|\left|\nabla w_{2}\right| \\
& \leq\left\|\nabla w_{1}\right\|_{L^{2}\left(\mathbb{T}^{n}\right)}\left\|\nabla w_{2}\right\|_{L^{2}\left(\mathbb{T}^{n}\right)} \\
& \leq\left\|w_{1}\right\|_{W^{1,2}\left(\mathbb{T}^{n}\right)}\left\|w_{2}\right\|_{W^{1,2}\left(\mathbb{T}^{n}\right)} .
\end{aligned}
$$

Using the Poincaré inequality, we have

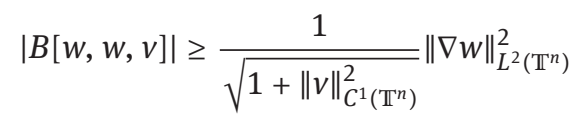

$$
\begin{aligned}
& \geq \frac{1}{\sqrt{1+\|v\|_{C^{1}\left(\mathbb{T}^{n}\right)}^{2}}\|\nabla w\|_{W^{1,2}\left(\mathbb{T}^{n}\right)}^{2}} .
\end{aligned}
$$

By (2.1), (2.2), and the Lax-Milgram theorem, for any $H \in L_{\mathrm{ave}}^{2}\left(\mathbb{T}^{n}\right)$, there exists a unique function

$$
u \in W_{\text {ave }}^{1,2}\left(\mathbb{T}^{n}\right)
$$

such that

$$
\int_{\mathbb{T}^{n}} \frac{\nabla u \cdot \nabla \psi}{\sqrt{1+|\nabla v|^{2}}}=\int_{\mathbb{T}^{n}} H \psi
$$

for all $\psi \in W_{\text {ave }}^{1,2}\left(\mathbb{T}^{n}\right)$. For any $\phi \in W^{1,2}\left(\mathbb{T}^{n}\right)$, we define $c_{\phi}:=\int_{\mathbb{T}^{n}} \phi$ and $\tilde{\phi}:=\phi-c_{\phi} \in W_{\text {ave }}^{1,2}\left(\mathbb{T}^{n}\right)$. By $(2.3)$ and $H \in L_{\text {ave }}^{2}\left(\mathbb{T}^{n}\right)$, we obtain

$$
\int_{\mathbb{T}^{n}} \frac{\nabla u \cdot \nabla \phi}{\sqrt{1+|\nabla v|^{2}}}=\int_{\mathbb{T}^{n}} \frac{\nabla u \cdot \nabla \tilde{\phi}}{\sqrt{1+|\nabla v|^{2}}}=\int_{\mathbb{T}^{n}} H \tilde{\phi}=\int_{\mathbb{T}^{n}} H \phi .
$$

Thus, Theorem 2.1 follows.

We define a mollifier as follows:

$$
\eta(x):= \begin{cases}C \exp \left(\frac{1}{|x|^{2}-1}\right) & \text { for }|x|<1, \\ 0 & \text { for }|x| \geq 1,\end{cases}
$$

where the constant $C>0$ is selected such that $\int_{\mathbb{R}^{n+1}} \eta=1$. We define

$$
\eta_{\lambda}(x):=\frac{1}{\lambda^{n}} \eta\left(\frac{x}{\lambda}\right)
$$

For any $f \in L^{2}\left(\mathbb{T}^{n} \times(-1,1)\right)$ and $x^{n+1} \in(-1+\lambda, 1-\lambda)$,

$$
\begin{aligned}
f_{\lambda}\left(x, x^{n+1}\right): & =\int_{\mathbb{T}^{n} \times(-1,1)} \eta_{\lambda}\left(x-y, x^{n+1}-y^{n+1}\right) f\left(y, y^{n+1}\right) d y \\
& =\int_{B^{n+1}(0, \lambda)} \eta_{\lambda}\left(y, y^{n+1}\right) f\left(x-y, x^{n+1}-y^{n+1}\right) d y,
\end{aligned}
$$


where $B^{n+1}(x, \lambda)$ is an open ball with center $x$ and radius $\lambda$ in $\mathbb{T}^{n} \times \mathbb{R}$. Moreover, for any

$$
g \in W^{1, p}\left(\mathbb{T}^{n} \times(-1,1) ; \mathbb{R}^{n+1}\right),
$$

we define $g_{\lambda}:=\left(g_{\lambda}^{1}, \ldots, g_{\lambda}^{n}, g_{\lambda}^{n+1}\right)=\left(g_{\lambda}^{\prime}, g_{\lambda}^{n+1}\right)$.

Lemma 2.2. Fix $\beta_{1}>0$ and $0<\lambda<1$. Suppose $v \in C^{1}\left(\mathbb{T}^{n}\right)$ satisfies $\|v\|_{C^{1}\left(\mathbb{T}^{n}\right)}<\beta_{1}$, and

$$
g \in W^{1, p}\left(\mathbb{T}^{n} \times(-1,1) ; \mathbb{R}^{n+1}\right)
$$

satisfies

$$
\partial_{n+1} g^{n+1}\left(x, x^{n+1}\right)>\beta_{1}\left|\partial_{n+1} g^{\prime}\left(x, x^{n+1}\right)\right| .
$$

For any positive constant $c_{0}>0$, if $v\left(\mathbb{T}^{n}\right)+c_{0} \subset(-1+\lambda, 1-\lambda)$, then

$$
\int_{\mathbb{T}^{n}} v(\nabla v) \cdot g_{\lambda}(x, v)<\int_{\mathbb{T}^{n}} v(\nabla v) \cdot g_{\lambda}\left(x, v+c_{0}\right) .
$$

Proof. From the assumptions, we compute

$$
\begin{aligned}
\int_{\mathbb{T}^{n}} v(\nabla v) & \cdot\left(g_{\lambda}\left(x, v+c_{0}\right)-g_{\lambda}(x, v)\right) \\
& =\int_{\mathbb{T}^{n}} \frac{1}{\sqrt{1+|\nabla v|^{2}}} \int_{v}^{v+c_{0}}-\nabla v \cdot \partial_{n+1} g_{\lambda}^{\prime}(x, t)+\partial_{n+1} g_{\lambda}^{n+1}(x, t) d t \\
& \geq \int_{\mathbb{T}^{n}} \frac{1}{\sqrt{1+|\nabla v|^{2}}} \int_{v}^{v+c_{0}}-\beta_{1}\left|\partial_{n+1} g_{\lambda}^{\prime}(x, t)\right|+\partial_{n+1} g_{\lambda}^{n+1}(x, t) d t \\
& \geq \int_{\mathbb{T}^{n}} \frac{1}{\sqrt{1+|\nabla v|^{2}}} \int_{v}^{v+c_{0}} \int_{\mathbb{T}^{n} \times(-1,1)} \eta_{\lambda}\left(x-y, t-y_{n+1}\right)\left\{-\beta_{1}\left|\partial_{n+1} g^{\prime}\left(y, y_{n+1}\right)\right|+\partial_{n+1} g^{n+1}\left(y, y_{n+1}\right)\right\} d t \\
& >0 .
\end{aligned}
$$

Lemma 2.2 follows.

Lemma 2.3. Suppose $g \in W^{1, p}\left(\mathbb{T}^{n} \times(-1,1)\right.$ and $v \in C^{1}\left(\mathbb{T}^{n}\right)$ with $\|v\|_{C^{1}\left(\mathbb{T}^{n}\right)} \leq \frac{7}{16}$. Let $q=\frac{n p}{n+1-p}$. Then there exists a constant $c_{1}=c_{1}(n, p)>0$ such that, if $\lambda<\frac{1}{8}$,

$$
\left\|g_{\lambda}(\cdot, v(\cdot))\right\|_{L^{q}\left(\mathbb{T}^{n}\right)} \leq c_{1}\|g\|_{W^{1, p}\left(\mathbb{T}^{n} \times(-1,1)\right)} .
$$

Proof. By the same proof as in [13, Lemma 2.3], we obtain

$$
\left\|g_{\lambda}(\cdot, v(\cdot))\right\|_{L^{q}\left(\mathbb{T}^{n}\right)} \leq c_{2}\left\|g_{\lambda}\right\|_{W^{1, p}\left(\mathbb{T}^{n} \times\left(-\frac{7}{8}, \frac{7}{8}\right)\right)},
$$

where $c_{2}=c_{2}(n, p)>0$. Using the Hölder inequality, we obtain

$$
\begin{aligned}
\int_{\mathbb{T}^{n} \times\left(-\frac{7}{8}, \frac{7}{8}\right)}\left|g_{\lambda}\right|^{p} d x & \leq \int_{\mathbb{T}^{n} \times\left(-\frac{7}{8}, \frac{7}{8}\right)}\left(\int_{B^{n+1}(x, \lambda)} \eta_{\lambda}^{1-\frac{1}{p}+\frac{1}{p}}\left(x-y, x^{n+1}-y^{n+1}\right)\left|g\left(y, y^{n+1}\right)\right| d y\right)^{p} d x \\
& \leq \int_{\mathbb{T}^{n} \times\left(-\frac{7}{8}, \frac{7}{8}\right)}\left(\int_{B^{n+1}(x, \lambda)} \eta_{\lambda}\left(x-y, x^{n+1}-y^{n+1}\right)\left|g\left(y, y^{n+1}\right)\right|^{p} d y\right) d x \\
& \leq \int_{\mathbb{T}^{n} \times(-1,1)}\left|g\left(y, y^{n+1}\right)\right|^{p}\left(\int_{B^{n+1}(y, \lambda)} \eta_{\lambda}\left(x-y, x^{n+1}-y^{n+1}\right) d x\right) d y \\
& =\int_{\mathbb{T}^{n} \times(-1,1)}\left|g\left(y, y^{n+1}\right)\right|^{p} d y .
\end{aligned}
$$

We can show that

$$
\left\|\nabla g_{\lambda}\right\|_{L^{p}\left(\mathbb{T}^{n} \times\left(-\frac{7}{8}, \frac{7}{8}\right)\right)} \leq\|\nabla g\|_{L^{p}\left(\mathbb{T}^{n} \times(-1,1)\right)}
$$

in the exact same manner, and Lemma 2.3 follows by (2.4) and (2.5). 
Theorem 2.4. Suppose $v \in C^{1}\left(\mathbb{T}^{n}\right)$ and

$$
g \in W^{1, p}\left(\mathbb{T}^{n} \times(-1,1) ; \mathbb{R}^{n+1}\right) .
$$

Then there exist constants $\varepsilon_{2}=\varepsilon_{2}(n, p)>0$ such that, if $\lambda<\frac{1}{8}, \varepsilon<\varepsilon_{2}$, and $\|v\|_{C^{1}\left(\mathbb{T}^{n}\right)} \leq \varepsilon^{1 / 2}$, then $g$ satisfies (1.2)-(1.4). Then there exist a unique function $u \in W_{\text {ave }}^{1,2}\left(\mathbb{T}^{n}\right)$ and a unique constant $-\frac{1}{4}<c_{v}<\frac{1}{4}$ such that

$$
\int_{\mathbb{T}^{n}} \frac{\nabla u \cdot \nabla \phi}{\sqrt{1+|\nabla v|^{2}}}=\int_{\mathbb{T}^{n}} v(\nabla v) \cdot g_{\lambda}\left(x, v+c_{v}\right) \phi
$$

for all $\phi \in W^{1,2}\left(\mathbb{T}^{n}\right)$.

Proof. We define

$$
F(t):=\int_{\mathbb{T}^{n}} v(\nabla v) \cdot g_{\lambda}(x, v+t) .
$$

The function $F$ is continuous. Suppose that $\varepsilon<\frac{1}{16^{2}}$. We will consider that the domain of $F$ is $\left[-\frac{1}{4}, \frac{1}{4}\right]$. By the mean value theorem, there exists a constant $c_{3}=c_{3}(n, p)>0$ such that

$$
\begin{aligned}
F\left(\frac{1}{4}\right) & =\int_{\mathbb{T}^{n}}(v(\nabla v)-v(0)+v(0)) \cdot g_{\lambda}\left(x, v+\frac{1}{4}\right) \\
& \geq-c_{3}\|v\|_{C^{1}\left(\mathbb{T}^{n}\right)}\left\|g_{\lambda}\left(\cdot, v(\cdot)+\frac{1}{4}\right)\right\|_{L^{q}\left(\mathbb{T}^{n)}\right.}+\int_{\mathbb{T}^{n}} g_{\lambda}^{n+1}\left(x, v+\frac{1}{4}\right) .
\end{aligned}
$$

By Lemma 2.3 and $\left\|v+\frac{1}{4}\right\|_{C^{1}\left(\mathbb{T}^{n}\right)} \leq \frac{5}{16}$, we obtain

$$
\left\|g_{\lambda}\left(\cdot, v(\cdot)+\frac{1}{4}\right)\right\|_{L^{q}\left(\mathbb{T}^{n}\right)} \leq c_{1}\|g\|_{W^{1, p}\left(\mathbb{T}^{n} \times(-1,1)\right)} .
$$

By (1.3) and (1.4), there exists a constant $c_{4}=c_{4}(n)>0$ such that

$$
\begin{aligned}
\int_{\mathbb{T}^{n}} g_{\lambda}^{n+1}\left(x, v+\frac{1}{4}\right) & =\int_{\mathbb{T}^{n}} \int_{B^{n+1}(0, \lambda)} \eta_{\lambda}\left(y, y^{n+1}\right) g^{n+1}\left(x-y, v+\frac{1}{4}-y^{n+1}\right) d y d x \\
& >\int_{\mathbb{T}^{n}} \int_{B^{n+1}(0, \lambda)} \eta_{\lambda}\left(y, y^{n+1}\right) g^{n+1}\left(x-y, \frac{1}{16}\right) d y d x \\
& >\int_{\mathbb{T}^{n}} \int_{B^{n+1}(0, \lambda)} \eta_{\lambda}\left(y, y^{n+1}\right)\left(g^{n+1}(x-y, 0)+\frac{\varepsilon}{16}\right) d y d x \\
& >\frac{c_{4}}{16} \varepsilon .
\end{aligned}
$$

By (1.2), (2.7)-(2.8), and $\|v\|_{C^{1}\left(\mathbb{T}^{n}\right)}<\varepsilon^{1 / 2}$, if

$$
\varepsilon<\left(\frac{c_{4}}{16 c_{1} c_{3}}\right)^{6}=: \varepsilon_{2}(n, p)
$$

then

$$
\begin{aligned}
F\left(\frac{1}{4}\right) & >-c_{1} c_{3}\|v\|_{C^{1}\left(\mathbb{T}^{n}\right)}\left\|g_{\lambda}\right\|_{W^{1, p}\left(\mathbb{T}^{n} \times(-1,1)\right)}+\frac{c_{4}}{16} \varepsilon \\
& >-c_{1} c_{3} \varepsilon^{\frac{7}{6}}+\frac{c_{4}}{16} \varepsilon \\
& >\varepsilon\left(-c_{1} c_{3} \varepsilon^{\frac{1}{6}}+\frac{c_{4}}{16}\right) \\
& >0 .
\end{aligned}
$$

Similarly, we can show that $F\left(-\frac{1}{4}\right)<0$. By Lemma 2.2 and the mean value theorem, there exists a unique constant $-\frac{1}{4}<c_{v}<\frac{1}{4}$ that satisfies $F\left(c_{v}\right)=0$. By using Theorem 2.1, Theorem 2.4 follows.

Let us define an operator $T: \mathcal{A}(s) \rightarrow W_{\text {ave }}^{1,2}\left(\mathbb{T}^{n}\right) \times\left[-\frac{1}{4}, \frac{1}{4}\right]$ by $T(v)=\left(T_{1}(v), T_{2}(v)\right):=\left(u, c_{v}\right)$ that satisfies (2.6), where

$$
\mathcal{A}(s):=\left\{w \in W_{\text {ave }}^{2, q}\left(\mathbb{T}^{n}\right):\|w\|_{W^{2, q}\left(\mathbb{T}^{n}\right)} \leq s\right\} .
$$


Theorem 2.5. There exist constants $\varepsilon_{3}=\varepsilon_{3}(n, p)>0$ and $c_{5}=c_{5}(n, p)>0$ such that, if $\lambda<\frac{1}{8}, \varepsilon<\min \left\{\varepsilon_{2}, \varepsilon_{3}\right\}$, $v \in \mathcal{A}\left(\varepsilon^{1 / 2}\right)$, and $g \in W^{1, p}\left(\mathbb{T}^{n} \times(-1,1) ; \mathbb{R}^{n+1}\right)$ satisfies (1.2)-(1.4), then

$$
\left\|T_{1}(v)\right\|_{W^{2, q}\left(\mathbb{T}^{n}\right)} \leq c_{5}\|g\|_{W^{1, p}\left(\mathbb{T}^{n} \times(-1,1)\right)} .
$$

Proof. We first assume that $v \in C^{\infty}\left(\mathbb{T}^{n}\right) \cap \mathcal{A}\left(\varepsilon^{1 / 2}\right)$. Using [6, Corollary 8.11], we obtain $T_{1}(v) \in C^{\infty}\left(\mathbb{T}^{n}\right)$. Thus, we can rewrite (2.6) as

$$
\frac{\Delta T_{1}(v)}{\sqrt{1+|\nabla v|^{2}}}+\nabla T_{1}(v) \cdot \nabla\left(\frac{1}{\sqrt{1+|\nabla v|^{2}}}\right)=-v(\nabla v) \cdot g_{\lambda}\left(x, v+T_{2}(v)\right) .
$$

Using [6, Theorem 9.11], we find that there exists a constant $c_{6}=c_{6}(n, p)>0$ such that

$$
\begin{gathered}
\left\|T_{1}(v)\right\|_{W^{2, q}\left(\mathbb{T}^{n}\right) \leq c_{6}}\left(\left\|T_{1}(v)\right\|_{L^{q}\left(\mathbb{T}^{n}\right)}+\left\|v(\nabla v) \cdot g_{\lambda}\left(x, v+T_{2}(v)\right)\right\|_{L^{q}\left(\mathbb{T}^{n}\right)}\right. \\
\left.+\left\|\nabla T_{1}(v) \cdot \nabla\left(\frac{1}{\sqrt{1+|\nabla v|^{2}}}\right)\right\|_{L^{q}\left(\mathbb{T}^{n}\right)}\right) .
\end{gathered}
$$

Using Lemma 2.3, we obtain

$$
\left\|v(\nabla v) \cdot g_{\lambda}\left(x, v+T_{2}(v)\right)\right\|_{L^{q}\left(\mathbb{T}^{n}\right)} \leq c_{1}\|g\|_{W^{1, p}\left(\mathbb{T}^{n} \times(-1,1)\right)} .
$$

Using the Sobolev inequality, we find that there exists a constant $c_{7}=c_{7}(n, p)>0$ such that

$$
\begin{aligned}
\left\|\nabla T_{1}(v) \cdot \nabla\left(\frac{1}{\sqrt{1+|\nabla v|^{2}}}\right)\right\|_{L^{q}\left(\mathbb{T}^{n}\right)} & \leq\left\|T_{1}(v)\right\|_{C^{1}\left(\mathbb{T}^{n}\right)}\left\|\nabla\left(\frac{1}{\sqrt{1+|\nabla v|^{2}}}\right)\right\|_{L^{q}\left(\mathbb{T}^{n}\right)} \\
& \leq c_{7}\left\|T_{1}(v)\right\|_{W^{2, q}\left(\mathbb{T}^{n}\right)}\|v\|_{W^{2, q}\left(\mathbb{T}^{n}\right)} .
\end{aligned}
$$

Next, we estimate the term $\left\|T_{1}(v)\right\|_{L^{q}\left(\mathbb{T}^{n}\right)}$. If $q \leq 2$, then, by (2.2) and Lemma 2.3, we obtain

$$
\begin{aligned}
\left\|T_{1}(v)\right\|_{L^{q}\left(\mathbb{T}^{n}\right)} & \leq c_{8}(n, p)\left\|T_{1}(v)\right\|_{L^{2}\left(\mathbb{T}^{n}\right)} \\
& \leq c_{9}(n, p) B\left[T_{1}(v), T_{1}(v), v\right]^{\frac{1}{2}} . \\
& =c_{9}\left(\int_{\mathbb{T}^{n}} \frac{\nabla T_{1}(v) \cdot \nabla T_{1}(v)}{\sqrt{1+|\nabla v|^{2}}}\right)^{\frac{1}{2}} \\
& =c_{9}\left(\int_{\mathbb{T}^{n}} v(\nabla v) \cdot g_{\lambda}\left(x, v+T_{2}(v)\right) T_{1}(v)\right)^{\frac{1}{2}} \\
& \leq c_{10}(n, p)\|g\|_{W^{1, p}\left(\mathbb{T}^{n}\right)}^{\frac{1}{2}}\left\|T_{1}(v)\right\|_{L^{\infty}\left(\mathbb{T}^{n}\right)}^{\frac{1}{2}} \\
& \leq c_{11}(n, p)\|g\|_{W^{1, p}\left(\mathbb{T}^{n}\right)}+\frac{1}{4 c_{6}}\left\|T_{1}(v)\right\|_{W^{2, q}\left(\mathbb{T}^{n}\right)} .
\end{aligned}
$$

If $q>2$, by (2.12) and the Riesz-Thorin theorem, we obtain

$$
\begin{aligned}
\left\|T_{1}(v)\right\|_{L^{q}\left(\mathbb{T}^{n}\right)} & \leq\left\|T_{1}(v)\right\|_{L^{2}\left(\mathbb{T}^{n}\right)}^{\frac{1}{q}}\left\|T_{1}(v)\right\|_{L^{2}\left(\mathbb{T}^{n}\right)}^{1-\frac{1}{q}} \\
& \leq c_{12}(n, p)\|g\|_{W^{1, p}\left(\mathbb{T}^{n}\right)}^{\frac{1}{2 q}}\left\|T_{1}(v)\right\|_{L^{\infty}\left(\mathbb{T}^{n}\right)}^{\frac{1}{2 q}+1-\frac{1}{q}} \\
& \leq c_{13}(n, p)\|g\|_{W^{1, p}\left(\mathbb{T}^{n}\right)}+\frac{1}{4 C_{6}}\left\|T_{1}(v)\right\|_{W^{2, q}\left(\mathbb{T}^{n}\right)} .
\end{aligned}
$$

By (2.9)-(2.13), there exists a constant $c_{14}=c_{14}(n, p)>0$ such that

$$
\left\|T_{1}(v)\right\|_{W^{2, q}\left(\mathbb{T}^{n}\right)} \leq c_{14}\left(\|g\|_{W^{1, p}\left(\mathbb{T}^{n} \times(-1,1)\right)}+\left\|T_{1}(v)\right\|_{W^{2, q}\left(\mathbb{T}^{n}\right)}\|v\|_{W^{2, q}\left(\mathbb{T}^{n}\right)}\right)+\frac{1}{4}\left\|T_{1}(v)\right\|_{W^{2, q}\left(\mathbb{T}^{n}\right)} .
$$

If $\varepsilon<\frac{1}{16 c_{14}^{2}}$, we obtain

$$
\left\|T_{1}(v)\right\|_{W^{2, q}\left(\mathbb{T}^{n}\right)} \leq 2 c_{14}\|g\|_{W^{1, p}\left(\mathbb{T}^{n} \times(-1,1)\right)} .
$$


For the general case of $v \in W^{2, q}\left(\mathbb{T}^{n}\right)$, suppose that $\left\{v_{m}\right\}_{m \in \mathbb{N}} \in C^{\infty}\left(\mathbb{T}^{n}\right)$ converges to $v$ in the sense of $C^{1}\left(\mathbb{T}^{n}\right)$. By (2.14), there exists a subsequence

$$
\left\{v_{m_{k}}\right\}_{k \in \mathbb{N}} \subset\left\{v_{m}\right\}_{m \in \mathbb{N}}
$$

such that $T_{1}\left(v_{m_{k}}\right)$ converges to a function $w_{\infty} \in W^{2, q}\left(\mathbb{T}^{n}\right)$ in the sense of $C^{1}\left(\mathbb{T}^{n}\right)$, and $T_{2}\left(v_{m_{k}}\right)$ converges to a constant $d_{\infty} \in\left[-\frac{1}{4}, \frac{1}{4}\right]$. For any $\phi \in W^{1,2}\left(\mathbb{T}^{n}\right)$, we obtain

$$
\begin{aligned}
& \int_{\mathbb{T}^{n}} v(\nabla v) \cdot g_{\lambda}\left(x, v+d_{\infty}\right) \phi-v\left(\nabla v_{m_{k}}\right) \cdot g_{\lambda}\left(x, v_{m_{k}}+T_{2}\left(v_{m_{k}}\right)\right) \phi \\
& \leq \int_{\mathbb{T}^{n}}|\phi|\left|v(\nabla v)-v\left(\nabla v_{m_{k}}\right)\right|\left|g_{\lambda}\left(x, v_{m_{k}}+T_{2}\left(v_{m_{k}}\right)\right)\right|+\left.\int_{\mathbb{T}^{n}}|\phi|\right|_{v_{m_{k}}+T_{2}\left(v_{m_{k}}\right)} ^{v+d_{\infty}} \partial_{n+1} g_{\lambda}(x, s) \mid \\
& \quad \rightarrow 0 \quad(k \rightarrow \infty)
\end{aligned}
$$

and

$$
\begin{aligned}
& \int_{\mathbb{T}^{n}} \frac{\nabla w_{\infty} \cdot \nabla \phi}{\sqrt{1+|\nabla v|^{2}}}-\frac{\nabla T_{1}\left(v_{m_{k}}\right) \cdot \nabla \phi}{\sqrt{1+\left|\nabla v_{m_{k}}\right|^{2}}} \\
& \quad \leq \int_{\mathbb{T}^{n}} \frac{\left(\nabla w_{\infty}-\nabla T_{1}\left(v_{m_{k}}\right)\right) \cdot \nabla \phi}{\sqrt{1+|\nabla v|^{2}}}+\int_{\mathbb{T}^{n}}\left(\nabla T_{1}\left(v_{m_{k}}\right) \cdot \nabla \phi\right)\left(\frac{1}{\sqrt{1+|\nabla v|^{2}}}-\frac{1}{\sqrt{1+\left|\nabla v_{m_{k}}\right|^{2}}}\right) \\
& \quad \rightarrow 0 \quad(k \rightarrow \infty) .
\end{aligned}
$$

By (2.15) and (2.16), we obtain

$$
\begin{aligned}
\int_{\mathbb{T}^{n}} \frac{\nabla w_{\infty} \cdot \nabla \phi}{\sqrt{1+|\nabla v|^{2}}}-v(\nabla v) \cdot g_{\lambda}\left(x, v+d_{\infty}\right) \phi \\
\quad=\lim _{k \rightarrow \infty} \int_{\mathbb{T}^{n}} \frac{\nabla T_{1}\left(v_{m_{k}}\right) \cdot \nabla \phi}{\sqrt{1+\left|\nabla v_{m_{k}}\right|^{2}}}-v\left(\nabla v_{m_{k}}\right) \cdot g_{\lambda}\left(x, v_{m_{k}}+T_{2}\left(v_{m_{k}}\right)\right) \phi \\
\quad=0,
\end{aligned}
$$

that is, $T(v)=\left(w_{\infty}, d_{\infty}\right)$. By (2.14) and (2.17), Theorem 2.5 follows.

Next, we provide the fixed-point theorem, which is needed later ([1, Theorem 1]). An operator $T: X \rightarrow A$ is considered weakly sequentially continuous if, for every sequence $\left\{x_{m}\right\}_{m \in \mathbb{N}} \subset X$ and $x_{\infty} \in X$ such that $x_{m}$ weakly converges to $x_{\infty}, T\left(x_{m}\right)$ weakly converges to $T\left(x_{\infty}\right)$.

Theorem 2.6. Let $X$ be a metrizable, locally convex topological vector space and let $\Omega$ be a weakly compact convex subset of $X$. Then any weakly sequentially continuous map $T: \Omega \rightarrow \Omega$ has a fixed point.

We first prove Theorem 1.1 in the case of $g_{\lambda}$.

Theorem 2.7. There exists a constant $\varepsilon_{4}=\varepsilon_{4}(n, p)>0$ such that, if $\lambda<\frac{1}{8}$ and $\varepsilon<\varepsilon_{4}$, then

$$
g \in W^{1, p}\left(\mathbb{T}^{n} \times(-1,1) ; \mathbb{R}^{n+1}\right)
$$

satisfies (1.2)-(1.4). Then there exists a function $u_{\lambda} \in W^{2, q}\left(\mathbb{T}^{n}\right)$ such that

$$
-\operatorname{div}\left(\frac{\nabla u_{\lambda}}{\sqrt{1+\left|\nabla u_{\lambda}\right|^{2}}}\right)=v\left(\nabla u_{\lambda}\right) \cdot g_{\lambda}\left(x, u_{\lambda}(x)\right) \quad \text { on } \mathbb{T}^{n} .
$$

Proof. The set $W^{2, q}\left(\mathbb{T}^{n}\right)$ is a metrizable, locally convex topological vector space, and the set $\mathcal{A}\left(\varepsilon^{1 / 2}\right)$ is a weakly compact convex subset of $W^{2, q}\left(\mathbb{T}^{n}\right)$. By (1.2) and Theorem 2.5, if $\varepsilon<\min \left\{\varepsilon_{2}, \varepsilon_{3}, c_{5}^{-6}\right\}=: \varepsilon_{4}$, we have

$$
\begin{aligned}
\left\|T_{1}(v)\right\|_{W^{2, q}\left(\mathbb{T}^{n}\right)} & \leq c_{5}\|g\|_{W^{1, p}\left(\mathbb{T}^{n} \times(-1,1)\right)} \\
& \leq c_{5} \varepsilon^{\frac{1}{6}} \varepsilon^{\frac{1}{2}} \\
& \leq \varepsilon^{\frac{1}{2}} \quad \text { for any } v \in \mathcal{A}\left(\varepsilon^{\frac{1}{2}}\right),
\end{aligned}
$$


that is, $T_{1}\left(\mathcal{A}\left(\varepsilon^{1 / 2}\right)\right) \subset \mathcal{A}\left(\varepsilon^{1 / 2}\right)$. Suppose that $\left\{v_{m}\right\}_{m \in \mathbb{N}}$ weakly converges to $v_{\infty}$ in the sense of $W^{2, q}\left(\mathbb{T}^{n}\right)$. According to Theorem 2.5, there exists a subsequence $\left\{v_{m_{k}}\right\}_{k \in \mathbb{N}} \subset\left\{v_{m}\right\}_{m \in \mathbb{N}}$ such that $T_{1}\left(v_{m_{k}}\right)$ weakly converges to a function $w_{\infty} \in W^{2, q}\left(\mathbb{T}^{n}\right)$ in the sense of $W^{2, q}\left(\mathbb{T}^{n}\right)$, and $T_{2}\left(v_{m_{k}}\right)$ converges to a constant $d_{\infty} \in\left[-\frac{1}{4}, \frac{1}{4}\right]$. By the same argument (2.15)-(2.17), for any $\phi \in W^{2, q}\left(\mathbb{T}^{n}\right)$,

$$
\int_{\mathbb{T}^{n}} \frac{\nabla w_{\infty} \cdot \nabla \phi}{\sqrt{1+\left|\nabla v_{\infty}\right|^{2}}}-v\left(\nabla v_{\infty}\right) \cdot g_{\lambda}\left(x, v_{\infty}+d_{\infty}\right) \phi=0,
$$

that is, we obtain $\lim _{k \rightarrow \infty} T_{1}\left(v_{m_{k}}\right)=T_{1}\left(v_{\infty}\right)$ by the uniqueness of solution of Theorem 2.4. Therefore, every convergent subsequence of $\left\{T_{1}\left(v_{m}\right)\right\}$ converges to $T_{1}\left(v_{\infty}\right)$, and $T_{1}$ is a weakly sequentially continuous map. Using Theorem 2.6, we obtain a function $v_{\lambda} \in W_{\text {ave }}^{2, q}\left(\mathbb{T}^{n}\right)$ satisfying

$$
-\operatorname{div}\left(\frac{\nabla v_{\lambda}}{\sqrt{1+\left|\nabla v_{\lambda}\right|^{2}}}\right)=v\left(\nabla v_{\lambda}\right) \cdot g_{\lambda}\left(x, v_{\lambda}(x)+T_{2}\left(v_{\lambda}\right)\right) \text { on } \mathbb{T}^{n},
$$

that is, $u_{\lambda}:=v_{\lambda}+T_{2}\left(v_{\lambda}\right) \in W^{2, q}\left(\mathbb{T}^{n}\right)$ satisfying (2.18).

Proof of Theorem 1.1. Suppose $u_{\lambda} \in W^{2, q}\left(\mathbb{T}^{n}\right)$ satisfies (2.18). By Theorem 2.5, there exists a convergent subsequence

$$
\left\{u_{\lambda_{k}}\right\}_{k \in \mathbb{N}} \subset\left\{u_{\lambda}\right\}_{0<\lambda<\frac{1}{8}}
$$

with a limit $u_{\infty} \in W^{2, q}\left(\mathbb{T}^{n}\right)$ in the sense of $C^{1}\left(\mathbb{T}^{n}\right)$ and $\lambda_{k} \rightarrow 0$. We show that $u_{\infty}$ satisfies (1.5). For any $\phi \in W^{1,2}\left(\mathbb{T}^{n}\right)$, we obtain

$$
\begin{aligned}
\int_{\mathbb{T}^{n}}-\operatorname{div}\left(\frac{\nabla u_{\lambda_{k}}}{\sqrt{1+\left|\nabla u_{\lambda_{k}}\right|^{2}}}-\frac{\nabla u_{\infty}}{\sqrt{1+\left|\nabla u_{\infty}\right|^{2}}}\right) \phi & =\int_{\mathbb{T}^{n}}\left(\frac{\nabla u_{\lambda_{k}}}{\sqrt{1+\left|\nabla u_{\lambda_{k}}\right|^{2}}}-\frac{\nabla u_{\infty}}{\sqrt{1+\left|\nabla u_{\infty}\right|^{2}}}\right) \cdot \nabla \phi \\
& \rightarrow 0 .
\end{aligned}
$$

Using Lemma 2.3, we have

$$
\begin{aligned}
& \int_{\mathbb{T}^{n}} v\left(\nabla u_{\lambda_{k}}\right) \cdot g_{\lambda_{k}}\left(x, u_{\lambda_{k}}\right)-v\left(\nabla u_{\infty}\right) \cdot g\left(x, u_{\infty}\right) \\
& =\int_{\mathbb{T}^{n}}\left(v\left(\nabla u_{\lambda_{k}}\right)-v\left(\nabla u_{\infty}\right)\right) \cdot g_{\lambda_{k}}\left(x, u_{\lambda_{k}}\right)+\int_{\mathbb{T}^{n}} v\left(\nabla u_{\infty}\right) \cdot\left(g_{\lambda_{k}}\left(x, u_{\lambda_{k}}\right)-g\left(x, u_{\lambda_{k}}\right)\right) \\
& \quad \quad \quad \int_{\mathbb{T}^{n}} v\left(\nabla u_{\infty}\right) \cdot\left(g\left(x, u_{\lambda_{k}}\right)-7 g\left(x, u_{\infty}\right)\right) \\
& =c_{1}\left\|v\left(\nabla u_{\lambda_{k}}\right)-v\left(\nabla u_{\infty}\right)\right\|_{C^{0}\left(\mathbb{T}^{n}\right)}\left\|g_{\lambda_{k}}\right\|_{W^{1, p}\left(\mathbb{T}^{n} \times(-1,1)\right)}+c_{1}\left\|g_{\lambda_{k}}-g_{\infty}\right\|_{W^{1, p}\left(\mathbb{T}^{n} \times(-1,1)\right)}+\iint_{\mathbb{T}^{n}}\left|\int_{u_{\infty}}^{u_{\lambda_{k}}} \partial_{n+1} g(x, s)\right| \\
& \rightarrow 0 .
\end{aligned}
$$

By (2.20) and (2.21), we obtain

$$
\begin{aligned}
\int_{\mathbb{T}^{n}}-\operatorname{div}( & \left.\frac{\nabla u_{\infty}}{\sqrt{1+\left|\nabla u_{\infty}\right|^{2}}}\right) \phi-v\left(\nabla u_{\infty}\right) \cdot g\left(x, u_{\infty}\right) \phi \\
\quad= & \lim _{k \rightarrow \infty} \int_{\mathbb{T}^{n}}-\operatorname{div}\left(\frac{\nabla u_{\lambda_{k}}}{\sqrt{1+\left|\nabla u_{\lambda_{k}}\right|^{2}}}\right) \phi-v\left(\nabla u_{\lambda_{k}}\right) \cdot g_{\lambda_{k}}\left(x, u_{\lambda_{k}}\right) \phi \\
& \rightarrow 0 .
\end{aligned}
$$

Thus, $u_{\infty}$ satisfies (1.5) using the fundamental lemma of the calculus of variations. By (2.19), we obtain

$$
\left\|u_{\infty}-\int_{\mathbb{T}^{n}} u_{\infty}(y) d y\right\|_{W^{2, q}\left(\mathbb{T}^{n}\right)} \leq \varepsilon^{\frac{1}{2}},
$$

and Theorem 1.1 follows. 


\section{References}

[1] O. Arino, S. Gautier and J.-P. Penot, A fixed point theorem for sequentially continuous mappings with application to ordinary differential equations, Funkcial. Ekvac. 27 (1984), no. 3, 273-279.

[2] T. Aubin, Some Nonlinear Problems in Riemannian Geometry, Springer Monogr. Math., Springer, Berlin, 1998.

[3] M. Bergner, The Dirichlet problem for graphs of prescribed anisotropic mean curvature in $\mathbb{R}^{n+1}$, Analysis (Munich) 28 (2008), no. 2, 149-166.

[4] D. Denny, A unique solution to a nonlinear elliptic equation, J. Math. Anal. Appl. 365 (2010), no. 2, 467-482.

[5] M. Feng, Periodic solutions for prescribed mean curvature Liénard equation with a deviating argument, Nonlinear Anal. Real World Appl. 13 (2012), no. 3, 1216-1223.

[6] D. Gilbarg and N. S. Trudinger, Elliptic Partial Differential Equations of Second Order, 2nd ed., Grundlehren Math. Wiss. 224, Springer, Berlin, 1983.

[7] W. Li and Z. Liu, Exact number of solutions of a prescribed mean curvature equation, J. Math. Anal. Appl. 367 (2010), no. 2, 486-498.

[8] S. Lu, Homoclinic solutions for a class of prescribed mean curvature Liénard equations, Adv. Difference Equ. 2014 (2014), Paper No. 244.

[9] T. Marquardt, Remark on the anisotropic prescribed mean curvature equation on arbitrary domains, Math. Z. 264 (2010), no. 3, 507-511.

[10] H. Pan, One-dimensional prescribed mean curvature equation with exponential nonlinearity, Nonlinear Anal. 70 (2009), no. 2, 999-1010.

[11] H. Pan and R. Xing, Time maps and exact multiplicity results for one-dimensional prescribed mean curvature equations. II, Nonlinear Anal. 74 (2011), no. 11, 3751-3768.

[12] Y. Tonegawa and Y. Tsukamoto, A diffused interface with the advection term in a Sobolev space, Calc. Var. Partial Differential Equations 59 (2020), no. 6, Paper No. 184.

[13] Y. Tsukamoto, The Dirichlet problem for a prescribed mean curvature equation, Hiroshima Math. J. 50 (2020), no. 3, 325-337.

[14] M. Zheng and J. Li, Nontrivial homoclinic solutions for prescribed mean curvature Rayleigh equations, Adv. Difference Equ. 2015 (2015), Paper No. 77. 